\title{
Melorheostosis and Osteopoikilosis Clinical and Molecular Description of an Italian Case Series
}

\author{
Maria Gnoli ${ }^{1}$ - Eric Lodewijk Staals ${ }^{2} \cdot$ Laura Campanacci $^{2} \cdot$ Maria Francesca Bedeschi $^{3} \cdot$ Flavio Faletra $^{4}$. \\ Salvatore Gallone ${ }^{5}$. Agostino Gaudio ${ }^{6} \cdot$ Teresa Mattina $^{7} \cdot$ Fiorella Gurrieri $^{8} \cdot$ Antonio Percesepe $^{9} \cdot$ Iria Neri $^{10}$. \\ Annalucia Virdi ${ }^{10} \cdot$ Morena Tremosini $^{1} \cdot$ Annamaria Milanesi $^{1} \cdot$ Evelise Brizola ${ }^{1}$. Elena Pedrini ${ }^{1} \cdot$ Luca Sangiorgi $^{11}$
}

Received: 18 March 2019 / Accepted: 16 May 2019 / Published online: 25 May 2019

(c) The Author(s) 2019

\begin{abstract}
Melorheostosis (MEL) is an uncommon, sclerosing disease, characterised by hyperostosis of long bones, resembling the flowing of candle wax. The disease is sporadic and the pathogenesis is still poorly understood. Occasionally, the same family can include individuals with MEL and Osteopoikilosis (OPK), a disease characterised by multiple round foci of increased bone density. LEMD3 gene mutations are related to OPK and Buschke-Ollendorff Syndrome, a genetic condition in which an association between MEL, OPK and skin lesions is observed. In rare cases, LEMD3 mutations and recently mosaic $M A P 2 K 1$ gene mutations have been correlated to MEL suggesting that somatic mosaicism could be causative of the disease. In this study, we described the clinical, radiological and molecular findings of 19 individuals with MEL and 8 with OPK and compared the results to the medical literature. The molecular analyses of this case series corroborate the available data in the medical literature, indicating that $L E M D 3$ germline mutations are not a major cause of isolated MEL and reporting five further cases of OPK caused by LEMD3 germline mutations.
\end{abstract}

Keywords Melorheostosis $\cdot$ Osteopoikilosis $\cdot$ LEMD3 gene $\cdot$ Osteopoikilosis with or without melorheostosis

\section{Introduction}

Melorheostosis (MEL, OMIM \#155950) [1] is an uncommon sclerosing bone disease characterised by "flowing hyperostosis", resembling the dripping of candle wax on the surface of the long bones. The distribution of the lesions

\section{Maria Gnoli}

maria.gnoli@ior.it

1 Department of Medical Genetics and Rare Orthopaedic Diseases, IRCCS Istituto Ortopedico Rizzoli, Via Pupilli 1, 40136 Bologna, Italy

2 3rd Clinic, Oncologic Orthopaedic Surgery, IRCCS Istituto Ortopedico Rizzoli, Bologna, Italy

3 Medical Genetics Unit, Fondazione IRCCS Ca'Granda Ospedale Maggiore Policlinico, Milan, Italy

4 Institute for Maternal and Child Health - IRCCS "Burlo Garofolo", Trieste, Italy

5 Clinic Neurogenetic Neuroscience Department, University of Turin, Turin, Italy

6 Department of Clinical and Experimental Medicine, University of Catania, Catania, Italy is usually unilateral and asymmetric or corresponds to a particular sclerotome $[1,2]$.

According to the literature, no gender prevalence is observed and the disease might be diagnosed at all ages [3]. However, most cases are diagnosed around the age of 20 years (50\% of cases) [3]. In childhood or adolescence,

7 Department BIOMETEC, University of Catania, Catania, Italy

8 Servizio di Genetica Medica, Fondazione Policlinico Universitario A. Gemelli IRCCS, Istituto di Medicina Genomica, Università Cattolica del Sacro Cuore, Rome, Italy

9 Medical Genetics - Department of Medicine and Surgery, University of Parma, Parma, Italy

10 Dermatology, Department of Experimental, Diagnostic and Specialty Medicine, Sant'Orsola-Malpighi Hospital, University of Bologna, Bologna, Italy

11 Department of Medical Genetics and Rare Orthopaedic Diseases \& CLIBI Laboratory, IRCCS Istituto Ortopedico Rizzoli, Bologna, Italy 
the progression of the bone lesions is variable and may lead to late diagnosis in adulthood [3]. There is a wide spectrum of clinical presentations, from asymptomatic cases to patients with chronic pain, joint stiffness, bone deformity, sometimes subcutaneous and soft tissues alterations [3-5].

The most frequently involved site is the appendicular skeleton (lower and upper limbs, hands and feet), with monostotic or polyostotic (or, rarely, bilateral) patterns [6]. Isolated MEL is usually a sporadic disease with no Mendelian pattern of inheritance or defined pathogenesis. The available medical literature includes mainly case reports or small case series, describing clinical aspects and surgical approaches, but the majority of those studies do not report any molecular investigation $[3,6,7]$.

Osteopoikilosis (OPK, OMIM \#166700) is an inherited disorder, with autosomal dominant pattern of transmission, characterised by dense bony island appearing as bright lesions on radiographs. It has an estimated incidence of $1: 50,000$ [8] and no gender differences. Since it is usually asymptomatic and only up to $20 \%$ of patients refers joint pain, the diagnosis of OPK is often an incidental finding on imaging studies performed for other health reasons [ 9 , $10]$.

OPK lesions can be either isolated, associated to MEL or part of Buschke-Ollendorff Syndrome (BOS, OMIM \#166700). BOS is an autosomal dominant connective tissue disorder characterised by skin and bone manifestations [1]. In the literature, one study describes individuals with OPK and other with MEL in the same family [11].

LEMD3 protein (MAN antigen 1 MAN1) acts as antagonist of TGF-B signalling, and loss of function by LEMD3 mutation can accelerate bone formation (OMIM *607844) [1, 2]. Germline mutations in LEMD3 gene have been related to a variable phenotypic spectrum, including isolated OPK, MEL with OPK and BOS [1,2]. Due to the presence of LEMD3 mutations in familial cases of MEL with OPK, the role of the gene in the pathogenesis of isolated MEL has been postulated $[5,11]$. Despite this, no germline mutations have been detected, and mosaic mutations were identified only in a few cases [12]. A recent study found no LEMD3 mutations with whole exome sequencing of 15 patients, instead mosaic mutations in $M A P 2 K 1$ gene were identified [13]. The importance of the MEK1-ERK1-2 in bone cell biology and in the disorder [13], as a role of somatic mutations of $K R A S$ gene, was advocated in the pathogenesis of the disease [2]. All these findings reinforce the hypothesis of mosaic mutation(s) as causative of MEL; nevertheless, further investigations are needed to confirm this.

In this study, we present a case series of 27 patients, 19 with MEL and 8 with OPK. This is the largest Italian study describing clinical and radiological findings with a LEMD3 molecular characterisation.

\section{Materials and Methods}

\section{Patients and Clinical Information}

All cases included in the study were selected according to clinical and radiological features of MEL or OPK. Patients of both gender and all ages were included. A study for clinical and molecular characterisation of skeletal rare diseases was approved by the Ethical Committee. Informed signed consent was obtained from all individual participants (or the parents in case of minors).

\section{Molecular Analysis}

Genomic DNA of all subjects was extracted from peripheral blood by using an automated workstation (Biomek NX, Beckman Coulter with Agencourt Genfind DNA isolation kit). DNA quality was checked with a NanoQuant Infinite M200 instrument (Tecan Group Ltd, Männedorf, Switzerland) before analyses.

In 3 MEL patients, LEMD3 genetic screening has been also performed on DNA from cultured fibroblasts (one) or from soft and bone tissue around the lesions obtained in surgical intervention for reduction of calcifications (2), extracted using QIAamp DNA Mini kit(QIAGEN GmbH, Hilden, Germany).

For each DNA sample, screening of all coding exons and flanking exon-intron junctions of LEMD3 was performed by Sanger sequencing. All regions were PCR-amplified using Veriti 96-well thermal cycler by Thermo Fisher Scientific in a 30- $\mu$ l volume. The results of amplification and the presence of right-sized PCR reaction products were confirmed by agarose gel electrophoresis. Amplification products were then sequenced in both forward and reverse direction using BigDye Terminator chemistry version 3.1 and ABI Prism 3130XL automated DNA sequencer (Thermo Fisher Scientific). The reference $L E M D 3$ sequence was obtained from GenBank accession numbers NM_014319.3.

All samples without any LEMD3 point mutation were analysed with the use of Real-Time PCR technique for the occurrence of exon, multiexon deletions and duplications. Q-PCR was performed on a Corbett Rotor Gene 6000 instrument using SYBR Green Dye I chemistry (RT2 Real-Time SYBR Green PCR Mix, SABiosciences, Frederick, MD, USA). Human b-actin (NC_000007.12) was used as endogenous control; DNA from healthy individuals was used as calibrator $(\mathrm{DDCt}=1$; DDCt for deleted exons $=0.5$; DDCt for duplicated exons $=2$ ). 


\section{Results}

Our series included 19 patients with diagnosis of MEL and 8 patients with diagnosis of OPK. Clinical data of MEL cases are described in Table 1.

\section{Isolated MEL Group}

This group included 19 patients and the diagnosis was performed based on of the presence of candle wax hyperostosis at the radiological examination (Fig. 1). The majority of the patients were females (13/19 patients) and the age at the moment of data collection was between 6 and 62 years (mean: 33 years), ranging from 14 to 62 years (mean: 35 years) in females and from 6 to 60 years in males (mean: 29 years).

Data about the first clinical manifestations were available for 15 individuals and are shown in Fig. 2a and b. Recurrent pain at the site of hyperostosis was reported as first clinical manifestation in eight patients (six females and two males). The presence of deformities was referred
Table 1 Clinical findings results and comparison to literature

\begin{tabular}{|c|c|c|}
\hline Clinical and radiological findings in MEL cases & $\begin{array}{l}\text { Number of patients } \\
\mathrm{n}(\%)\end{array}$ & $\begin{array}{l}\text { Smith et al. } \\
\mathrm{n}(\%)\end{array}$ \\
\hline Age at the first investigation/clinical manifestation & All cases $(18)$ & All cases $(23)$ \\
\hline 0 to 18 years & $5(28 \%)$ & \\
\hline$>18$ years & $3(72 \%)$ & \\
\hline Gender & All cases (19) & \\
\hline Female & $13(68.4)$ & $5(79.2)$ \\
\hline Male & $6(31.6)$ & $19(20.8)$ \\
\hline Affected site & All cases (19) & \\
\hline Head & 0 & $2(8.3)$ \\
\hline Spine & 0 & $4(16.6)$ \\
\hline Arm & $2(10.5)$ & $8(33.3)$ \\
\hline Hand or wrist & $4(21)$ & $7(29.1)$ \\
\hline Leg & $11(57.9)$ & $16(66.7)$ \\
\hline Ankle or foot & $5(26.3)$ & $9(37.5)$ \\
\hline Single limb & $18(94.7)$ & $20(83)$ \\
\hline Upper limb ${ }^{\mathrm{a}}$ & $1(5.3)$ & \\
\hline Lower limb ${ }^{b}$ & $10(52.6)$ & \\
\hline Only hand & $4(21)$ & \\
\hline Only foot & $3(15.8)$ & \\
\hline Both lower + upper limbs & $1(5.3)$ & \\
\hline Upper limb + hand & $5(26.3)$ & \\
\hline Lower limb + foot & $13(68.4)$ & \\
\hline Both lower and upper limb + hand and foot & $1(5.3)$ & \\
\hline Cause leading to the first investigation & All cases $(15)$ & \\
\hline Pain & $8(53)$ & $20(83.3)$ \\
\hline Deformity & $2(13.3)$ & $13(54.2)$ \\
\hline Functional limitation/stiffness & $3(20)$ & $11(45.8)$ \\
\hline Incidental diagnosis & $2(13.3)$ & \\
\hline \multicolumn{3}{|l|}{ Age at the moment of the evaluation } \\
\hline Mean & 32 & 36.5 \\
\hline Median & 32 & 41.5 \\
\hline Range & $6-62$ & $3-68$ \\
\hline Clinical findings at the moment of the evaluation & All cases (17) & \\
\hline Pain & $15(88)$ & \\
\hline Deformity & $6(35.2)$ & \\
\hline Functional limitation/stiffness & $8(47)$ & \\
\hline
\end{tabular}

${ }^{\mathrm{a}}$ Cases with hand only involvement excluded

${ }^{\mathrm{b}}$ Cases with foot only involvement excluded 


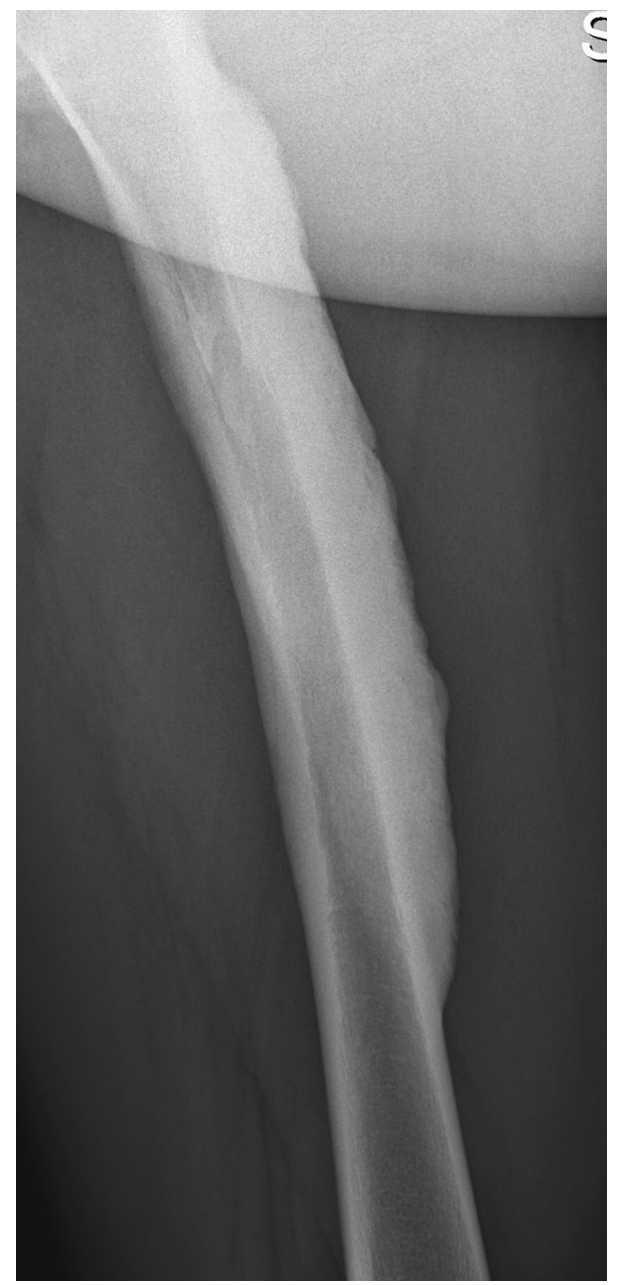

Fig. 1 Radiological image of a femur with candle wax hyperostosis

in two females, whereas a female and two males had limitations of joint motion or stiffness. Only two cases (a male and a female) (13\%) had an incidental diagnosis after $\mathrm{X}$-rays were performed for different clinical indications.

Considering the age of the first clinical manifestations, only two male patients had the first disease investigation already during childhood, while 13/18 patients (11 females

(a) First clinical manifestation in $\geq 18 y$

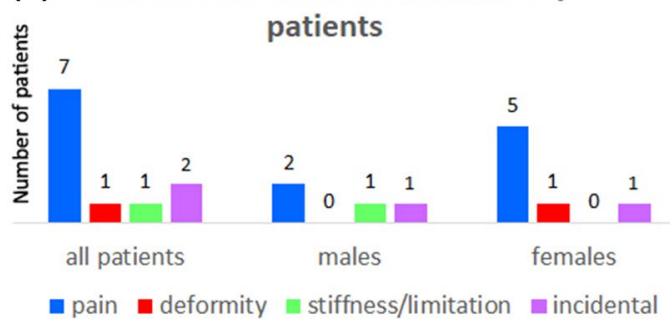

and 2 males) were adults; only three patients, all females, had the first manifestations in adolescence.

At the time of our evaluation, pain was the most frequently reported clinical manifestation, referred by 15 (11 females and 4 males) patients. Limitation of joint movement and/or stiffness was reported in 8 cases. Only 6 cases ( 5 females and a male) had some grade of deformity related to hyperostosis.

Figure 3 shows the limb involvement by hyperostosis in 19 MEL patients. In the majority of cases, (13 patients, 5 males and 8 females), the hyperostosis affected the lower limbs. This included one female with hyperostosis areas limited to the foot. In one case, the pelvic bones were also involved, and in another one both pelvis and lower limbs, including feet, were involved. Upper limbs, including wrists or hands, were affected in 5 patients. The hand is the second most frequently involved site (4/19 patients, all females); only one male patient had hyperostosis in the upper limb but not in the hands. Only one patient had hyperostosis in both upper and lower limbs.

Skin manifestations were observed in two females. Both of them had skin thickness; in particular, one patient had calcifications above the area of the skeletal lesion and the other one had a dyschromia. In this last case, molecular analysis was also performed on DNA from both cultured fibroblasts and bone tissue without detecting any LEMD3 mutation.

Connective tissue nevus or true sclerodermic phenotypes were not observed in our series.

No germline mutations in LEMD3 gene have been identified in this group of patients. In 3 cases, molecular analysis was performed also from tissue samples and no somatic mutations of $L E M D 3$ have been found in these samples.

\section{Osteopoikilosis Group}

Eight patients (six males and two females) had a radiological diagnosis of OPK (Fig. 4), four of them characterised by a positive family history for OPK. In this group, the mean age at our evaluation was 39 years (ranging from 16 to 53 years).

In one case, the onset of the disease was during childhood (about 10-year-old boy), in four patients during

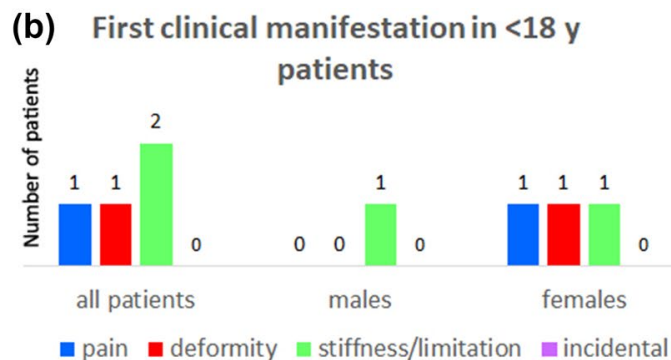

Fig. 2 a First clinical manifestation in patients aged $\geq 18$ years. b First clinical manifestation in patients aged $<18$ years 


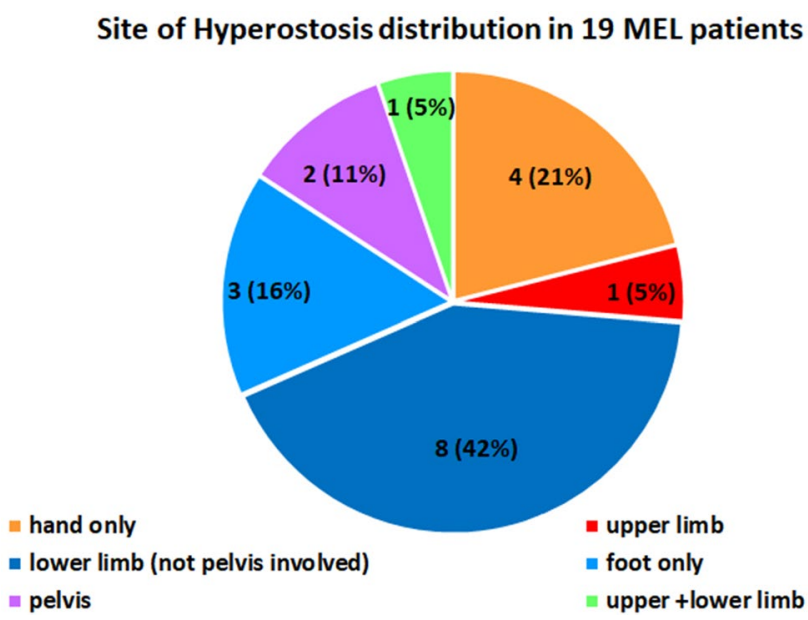

Fig. 3 Site of hyperostosis distribution in MEL cases

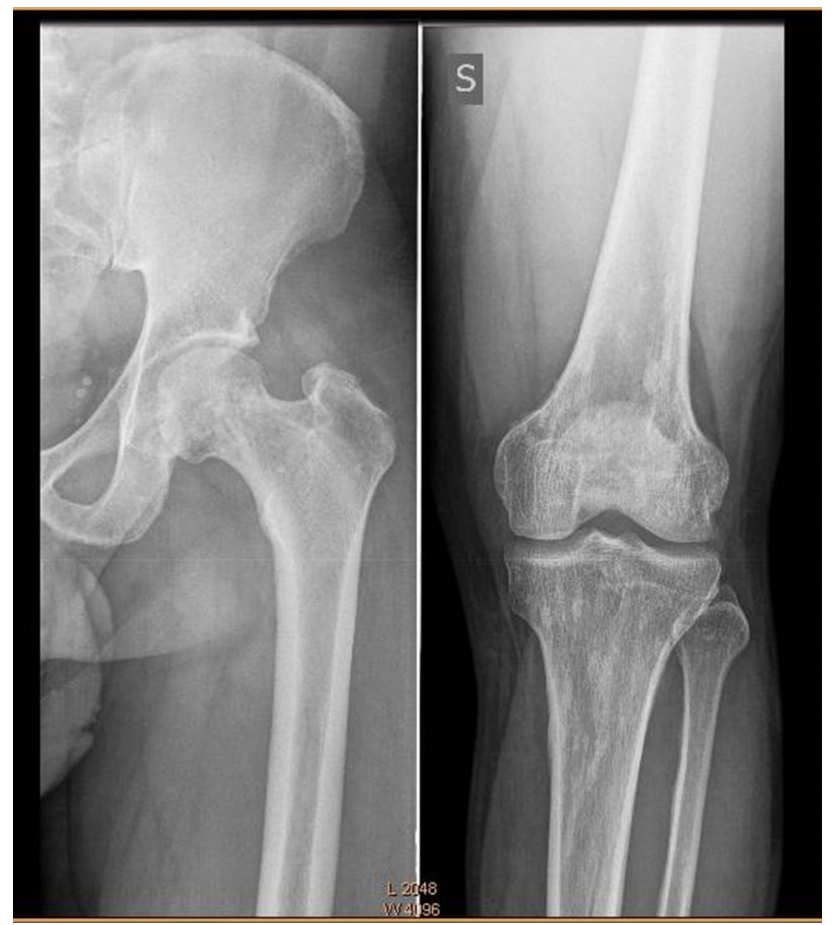

Fig. 4 X-ray showing hyperostotic spots on the lower limb of a patient with OPK

adulthood (three males and a female with an age ranging from 41 to 53 years) and 3 during puberty (two males and a female 14 to 16 years old).

In five patients (three males and two females), the first investigations were performed for recurrent pain, in two cases (both males) the diagnosis was incidental. In one asymptomatic case, the diagnosis was performed by molecular analysis for a positive family history.
At the moment of data collecting, hyperostotic areas were in both lower and upper limbs in four patients, in lower limbs in two patients and in the pelvis in three cases. At the time of clinical evaluation, four patients reported pain; bone deformities or joint limitations were present in one case, and stiffness in two patients.

LEMD3 molecular analysis identified a germline pathologic mutation in five among eight patients affected by OPK, belonging to seven different families. In particular, we detected a heterozygous deletion of the whole gene in a patient, a splice site mutation (c.1628-2A $>$ G) in a second one, a non-sense mutation (c.2032C > T; p.Arg678*) in a third one. The last two patients are relatives and carry a duplication (c.1754dup, p.Asn585Lysfs*15).

The patient carrying the whole gene deletion was an adult male with skin lesions; the clinical diagnosis was suspected during childhood after radiological investigation performed for lower limbs pain.

The splice site mutation c.1628-2A $>$ G was not previously described in the medical literature and we identified this mutation in a woman diagnosed for OPK in adult age after clinical investigations requested for lower limb joint pain. She presented OPK areas around the left knee.

The c.2032C > T variant (p.Arg678*) has been previously described by Couto et al. [11] in two families. In our study, in the patient carrying the $\mathrm{c} .2032 \mathrm{C}>\mathrm{T}$ mutation, the diagnosis was performed in adult age after evaluations for lower limbs pain. The radiographs showed hyperostotic spots typical of OPK in the lower limb (Fig. 4).

The last identified variant [c.1754dup mutation (p.Asn585Lysfs*15)] was not reported in main mutational databases (LOVD, Clinvar) to date and we found this mutation in two Italian patients (father and son) affected by OPK in the lower limb; the disease was accidentally identified during X-rays performed for a suspected traumatic fracture. In both cases, further investigations showed typical hyperostotic areas in hands, feet and pelvis.

\section{Discussion}

MEL and OPK are hyperostotic bone diseases, with clinical expression ranging from asymptomatic to recurrent pain, bone deformity or joint limitation. While the genetic basis of OPK has been defined, MEL pathogenesis is to this date largely unknown.

In this study, which includes the largest series of MEL patients in Italy evaluated for the presence of $L E M D 3$ variant until now, we did not detect any $L E M D 3$ germline mutation in the isolated MEL group.

The imaging appearance of both MEL and OPK is quite typical. Our results are consistent with literature, confirming that LEMD3 mutations do not seem to play a role in 
isolated MEL. On the other hand, we identified a LEMD3 pathological variant in 5 of 8 OPK patients. Thus, the radiological diagnosis of OPK seems to be consistently associated with LEMD3 mutations and autosomal dominant form of the disease.

Isolated MEL is a sporadic disease, but because few families with recurrence of MEL and/or OPK exist and the diagnosis can be incidental for the absence of clinical manifestation, molecular analysis can be the only tool to exclude an autosomal dominant form of the disease, even if this seems very rare.

Between our MEL cases, female gender was more prevalent (nearly 70\%), and similar to the ratio of $4: 1$ female to male found by Smith et al. [6]. As highlighted by Smith et al., this finding contrasts the mosaicism hypothesis that would have no impact on gender, since both genders can be equally affected without predilection, unless the candidate genes are located on chromosome X.

The clinical expression of MEL was quite variable in our case series. According to literature data, MEL can be diagnosed at any age, but approximately $50 \%$ of the diagnoses are made around the age of 20 years. We observed that the first clinical suspicion occurred for most cases during adulthood $(70 \%)$, and only about $30 \%$ of patients received the diagnosis before the adult age. A difference in the age of onset appears between the male and female groups: onethird of the MEL patients had the first clinical manifestation in adolescence and they are all females. Furthermore, it is known that the diagnosis of MEL or OPK is often incidental but in this series few cases of MEL (about 13\%) received the diagnosis after $\mathrm{X}$-rays performed for other indications: pain, bone deformities or joint limitations were the reasons to medical investigations in most of the patients.

The main clinical manifestation observed was recurrent pain, which is consistent with literature. Lower limbs were the most common site involved, confirming the data from the series reported by Smith et al. [6]; no skull involvement was found. The disorder is nearly always monomelic; in a previous report, more than $80 \%$ of the cases had a single limb involvement, as this series, where only one case showed the disease in both lower and upper limbs. In particular, the bilateral distribution of the lesions in one case and the involvement of the upper and lower right limb in another one were in contrast with the sclerotomal hypothesis.

Jha et al. observed the same pattern of distribution in MEL by CT analysis in only a minority of the cases, stressing the need for further studies to understand both aetiology and the causes for clinical variability in MEL [14].

In the eight patients with radiological diagnosis of OPK, we found a $L E M D 3$ pathological variant in four patients and a whole gene deletion in one (more than $60 \%$ of detection rate). No evident genotype-phenotype correlation could be performed. The c.2032C > T(p.Arg678*) LEMD3 mutation has been previously described in two families with OPK and related to the disease; one is a third-generation family of Azorean origin (three members) and the other one is a small family of two members from Ireland [12]. As in the case we described, the molecular diagnosis was performed in adult age for the first family. The second family's proband had the molecular diagnosis when she was 12 years old and showed OPK with MEL. From historical information and analysis of haplotype, the authors speculated a common founder in these two families.

The ethnic origin of the case reported here carrying the p.Arg678* mutation is in line with the origins of the population in Azores, that is composed by different populations [15]. OPK involved a lower limb and the diagnosis was performed after evaluation for lower limbs pain. X-ray showed hyperostosis spots in the lower limb, but not the typical candle wax appearance of MEL.

In addition, a mutation that was not previously reported in main mutational databases, c.1754dup (p. Asn585Lysfs*15), was identified in two related patients, a father and a son. Interestingly, both of them had an almost asymptomatic disease and had similar unilateral distribution of the lesions, in both upper and lower limbs.

In the medical literature, a smaller stature has been reported in adults with OPK. In our series, even if we do not know the parental target, patients affected by OPK had a height below $50^{\circ}$ percentile; in particular, three of them are around the $3^{\circ}$ percentile of the Italian population, according to literature data [16]. For the best of our knowledge, none of our OPK patients underwent evaluation of developmental delay, which previously has been reported in as high percentage as 50\% of patients with LEMD3 mutation [16].

A collecting bias should be considered in this study, since our department is part of a surgical orthopaedic institute. If the first manifestation is mild and related to skin involvement around the skeletal lesions-as described in medical literature, especially in OPK - this rare condition could not be suspected at the beginning of the clinical presentation and the diagnosis can be missed. This would lead to a diagnostic delay at a specialised centre for rare skeletal diseases; in particular, the patient may not remember when the first clinical signs began before the diagnosis of bone hyperostosis.

In conclusion, our clinical findings in the MEL and OPK series were in line with the medical literature, except for the low percentage of incidental diagnosis of MEL, that we found in only $10 \%$ of cases. A LEMD3 gene mutation has been found in more than $60 \%$ of OPK patients in our series. Instead, no alteration was identified in isolated MEL, confirming that other genes and mechanisms had to be involved in pathogenesis and variability of the disease. Thus, further investigations will be needed to clarify these aspects, that potentially will shed light into bone biology and therapies for other diseases affecting bone mass. 
Acknowledgements We thank all the patients and their families for their participation in this study and the colleagues who contribute to this work. We are grateful to BIOGEN biobank, member of the Telethon Network of Genetic Biobanks and of the EuroBioBank network, which provided us with the biological samples.

Author Contributions MG designed the study and prepared the first draft of the paper. She had the main role in revising process for the final draft. She was also involved in the patients' recruitment, in clinical diagnosis and in the collection, revision and organisation of all clinical data. ELS, LC, MFB, FF, SG, AG, TM, FG, AP, IN, AV, MT, $\mathrm{AM}, \mathrm{EB}$ and LS contributed to clinical diagnosis, collection of clinical data and draft of the paper. EP performed molecular analysis and interpretation of molecular results and draft of the paper. All authors revised the paper critically for intellectual content and then approved the final version. All authors agree to be accountable for the work and to ensure the accuracy and integrity of the paper. All authors read and approved the final manuscript.

\section{Compliance with Ethical Standards}

Conflict of interest Maria Gnoli, Eric Lodewijk Staals, Laura Campanacci, Maria Francesca Bedeschi, Flavio Faletra, Salvatore Gallone, Agostino Gaudio, Teresa Mattina, Fiorella Gurrieri, Antonio Percesepe, Iria Neri, Annalucia Virdi, Morena Tremosini, Annamaria Milanesi, Evelise Brizola, Elena Pedrini and Luca Sangiorgi declare that they have no conflict of interest.

Human and Animal Rights A study for clinical and molecular characterization of skeletal rare diseases (Prot n. 21623 : "BIOGEN"; "Istituzione di una Biobanca Genetica diagnostica e di ricerca presso la S.S.D. di Genetica Medica e Malattie Rare Ortopediche") and amendments (Prot. gen. 0041207 and following,) were approved by the Ethical Committee of Istituto Ortopedico Rizzoli.

Informed Consent Informed signed consent was obtained from all individual participants (or the parents in case of minors).

Open Access This article is distributed under the terms of the Creative Commons Attribution 4.0 International License (http://creativeco mmons.org/licenses/by/4.0/), which permits unrestricted use, distribution, and reproduction in any medium, provided you give appropriate credit to the original author(s) and the source, provide a link to the Creative Commons license, and indicate if changes were made.

\section{References}

1. Online Mendelian Inheritance in Man, OMIM® (2018) McKusick-Nathans Institute of Genetic Medicine, Johns Hopkins University; Baltimore: 2018 World Wide Web. http://omim.org. Accessed 1 Dec 2018

2. Whyte MP, Griffith M, Trani L, Mumm S, Gottesman GS, McAlister WH, Krysiak K, Lesurf R, Skidmore ZL, Campbell KM, Rosman IS, Bayliss S, Bijanki VN, Nenninger A, Van Tine BA, Griffith OL, Melorheostosis MER (2017) Exome sequencing of an associated dermatosis implicates postzygotic mosaicism of mutated KRAS. Bone 101:145-155. https://doi.org/10.1016/j. bone.2017.04.010

3. Kotwal A, Clarke BL (2017) Melorheostosis: a rare sclerosing bone dysplasia. Curr Osteoporos Rep 15(4):335-342. https://doi. org/10.1007/s11914-017-0375-y
4. Freyschmidt J (2001) Melorheostosis: a review of 23 cases. Eur Radiol 11(3):474-479

5. Hellemans J, Preobrazhenska O, Willaert A, Debeer P, Verdonk PC, Costa T, Janssens K, Menten B, Van Roy N, Vermeulen SJ, Savarirayan R, Van Hul W, Vanhoenacker F, Huylebroeck D, De Paepe A, Naeyaert JM, Vandesompele J, Speleman F, Verschueren K, Coucke PJ, Mortier GR (2004) Loss-of-function mutations in LEMD3 result in osteopoikilosis, Buschke-Ollendorff syndrome and melorheostosis. Nat Genet 36:1213-1218

6. Smith GC, Pingree MJ, Freeman LA, Matsumoto JM, Howe BM, Kannas SN, Pyfferoen MD, Struss LT, Wenger DE, Amrami KK, Matsumoto M, Jurisson ML (2017) Melorheostosis: a retrospective clinical analysis of 24 patients at the Mayo Clinic. PMR 9(3):283-288. https://doi.org/10.1016/j.pmrj.2016.07.530

7. Gagliardi GG, Mahan KT (2010) Melorheostosis: a literature review and case report with surgical considerations. J Foot Ankle Surg 49(1):80-85

8. Ng C, Schwartzman L, Moadel R, Haigentz M Jr (2015) Osteopoikilosis: a benign condition with the appearance of metastatic bone disease. J Clin Oncol 33(18):e77-e78. https://doi. org/10.1200/JCO.2013.50.5222

9. Tsai SY, Wang SY, Shiau YC, Wu YW (2016) Benign incidental findings of osteopoikilosis on Tc-99 m MDP bone SPECT/ CT: a case report and literature review. Medicine (Baltimore) 95(23):e3868

10. Boulet C, Madani H, Lenchik L, Vanhoenacker F, Amalnath DS, de Mey J, De Maeseneer M (2016) Sclerosing bone dysplasias: genetic, clinical and radiology update of hereditary and nonhereditary disorders. Br J Radiol 89(1062):20150349. https://doi. org/10.1259/bjr.20150349

11. Couto AR, Bruges-Armas J, Peach CA, Chapman K, Brown MA, Wordsworth BP, Zhang Y (2007) A novel LEMD3 mutation common to patients with osteopoikilosis with and without melorheostosis. Calcif Tissue Int 81(2):81-84

12. Zhang Y, Castori M, Ferranti G, Paradisi M, Wordsworth BP (2009) Novel and recurrent germline LEMD3 mutations causing Buschke-Ollendorff syndrome and osteopoikilosis but not isolated melorheostosis. Clin Genet 75(6):556-561. https://doi.org/10.111 1/j.1399-0004.2009.01177.x

13. Kang H, Jha S, Deng Z, Fratzl-Zelman N, Cabral WA, Ivovic A, Meylan F, Hanson EP, Lange E, Katz J, Roschger P, Klaushofer K, Cowen EW, Siegel RM, Marini JC, Bhattacharyya T (2018) Somatic activating mutations in MAP2K1 cause melorheostosis. Nat Commun 9(1):1390. https://doi.org/10.1038/s41467-01803720-z

14. Jha S, Laucis N, Kim L, Malayeri A, Dasgupta A, Papadakis GZ, Karantanas A, Torres M, Bhattacharyya T (2018) CT analysis of anatomical distribution of melorheostosis challenges the sclerotome hypothesis. Bone 117:31-36. https://doi.org/10.1016/j. bone.2018.09.005

15. Santos C, Lima M, Montiel R, Angles N, Pires L, Abade A, Aluja MP (2003) Genetic structure and origin of peopling in the Azores islands (Portugal): the view from mtDNA. Ann Hum Genet 67(Pt 5):433-456

16. Pope V, Dupuis L, Kannu P, Mendoza-Londono R, Sajic D, So J, Yoon G, Lara-Corrales I (2016) Buschke-Ollendorff syndrome: a novel case series and systematic review. Br J Dermatol 174(4):723-729. https://doi.org/10.1111/bjd.14366

Publisher's Note Springer Nature remains neutral with regard to jurisdictional claims in published maps and institutional affiliations. 